\title{
Assessment of the Psychological Comorbidity, Pathophysiological Mechanisms, and Treatment Implications in Patients with Chronic Orofacial Pain
}

\author{
Melissa L Mehalick ${ }^{1}$, John P Garofalo ${ }^{1 *}$, Celeste N Sanders ${ }^{2}$ and Robert J Gatchel ${ }^{2}$ \\ ${ }^{1}$ Department of Psychology, Washington State University Vancouver, 14204 NE Salmon Creek Ave, Vancouver, Washington, USA \\ ${ }^{2}$ Department of Psychology, University of Texas at Arlington, 701 South Nedderman Drive, Arlington, TX, USA
}

\begin{abstract}
Chronic orofacial pain is a multifaceted health problem that like many other forms ofchronic pain bears deleterious effects upon quality of life as well as psychological andphysiological well-being. Due to a poorly understood etiology, effective treatment strategies are lacking and tend to lack a guiding integrative conceptual framework toform the basis and development of intervention. This review seeks to provide an updatedreview of the comorbid psychological disorders and characteristics that are common among chronic orofacial pain patients, while also examining the pathophysiological mechanisms underlying orofacial pain. Rather than consider the emotional, cognitive, andneuroendocrine influences upon pain perception and severity individually, these factorsshould be viewed as working in concert with one another. It is this interplay amongst distinct psychological characteristics governing the patient along with physiologicalmechanisms that exacerbate the pain. Together, the goal is to identify unique characteristics surrounding orofacial pain and offer some plausible insights for effectivetreatment outcomes.
\end{abstract}

Keywords: Chronic orofacial pain; Temporomandibular joint; Periaqueductal gray

\section{Introduction}

Chronic Orofacial Pain (COP) represents a complex pain condition with an etiology comprised of many factors including those falling within biological, psychological, andsocial domains. The complexity of this condition underscores the urgency to evaluatethese domains from an integrative perspective rather than one that simply addresses thepotential etiological mechanisms in a singular fashion. Indeed, a review of the trajectory of this pain condition reveals a dynamic pathway in which numerous factors seeminglyplay a role in its manifestation, as well as its chronicity.

By definition, "chronic" orofacial pain represents a pain that has persisted for a period oftime that is longer than the believed recovery time for that body site to heal.Approximately $40-70 \%$ of the US reportedly demonstrate a symptom of orofacial pain, and $10 \%$ meet the criteria for having COP to the point that it is considered disabling andis significantly compromising activities of daily living [1]. The intention of this paper will not center on providing an exhaustive review, but instead, to highlight key featuresof COP from a biopsychosocial perspective. Specifically, the transition that lead sorofacial pain from the acute to the chronic stage will be reviewed by underscoring well established mechanisms believed to facilitate this transition. Embedded in this discussion, the psychophysiological mechanisms that are less established in the manifestation of COP will also be reviewed. These mechanisms may distinguish COP from other types ofpain. Much of the data presented stem from studies that have included a wide range of orofacial pain conditions including, but not limited to, temporomandibular disorders, trigeminal pain, and headaches.

\section{The Psychological Pathogenesis from Acute to Chronic Pain}

There are a number of factors that contribute to the transition from acute pain to chronicpain. It is suspected that the identification of psychological issues serves as an indicatorthat acute pain is becoming more chronic in nature $[2,3]$. This is logical in that chronicpain appears to be associated with diagnoses of psychological disorders (e.g., mooddisorders and anxiety disorders) [4,5] and other adverse psychological constructs whose presence has often been suspected in complicating the clinical picture of chronic pain. Asdepicted by the model below (Figure 1), these factors are intertwined with physiological and behavioral factors that further maintain the pain condition.

Gatchel et al. [6] propose an empirically supported model, consisting of threestages, which explain the transition by which acute pain evolves into chronic pain. According to this model, Stage 1 involves the emergence of acute stage of pain coupledwith the anticipated preoccupation that results from the emergence of the unpleasantstimulus. At this point, the individual with pain becomes

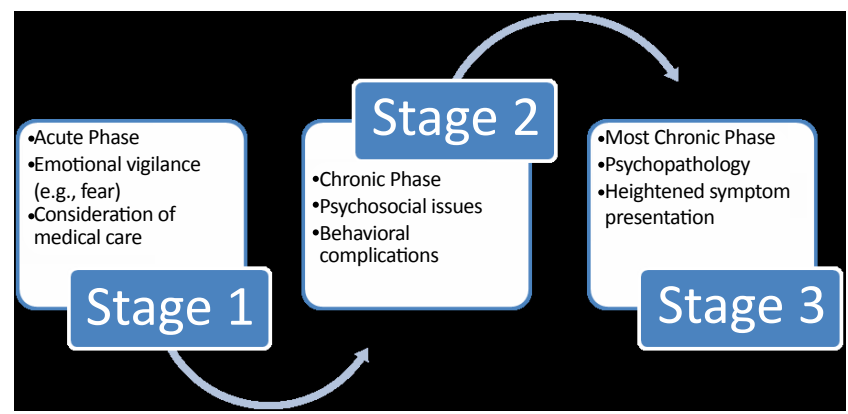

Figure 1: Development from acute to chronic pain.

*Corresponding author: John P Garofalo, PhD, Associate Professor/Program Director of Psychology, 14204 NE Salmon Creek Avenue, Vancouver, WA 98686 USA, E-mail: jgarofalo@vancouver.wsu.edu

Received May 02, 2013; Accepted June 06, 2013; Published June 16, 2013

Citation: Mehalick ML, Garofalo JP, Sanders CN, Gatchel RJ (2013) Assessment of the Psychological Comorbidity, Pathophysiological Mechanisms, and Treatment Implications in Patients with Chronic Orofacial Pain. Anaplastology S2: 001. doi:10.4172/2161-1173.S2-001

Copyright: (c) 2013 Mehalick ML, et al. This is an open-access article distributed under the terms of the Creative Commons Attribution License, which permits unrestricted use, distribution, and reproduction in any medium, provided the original author and source are credited. 
vigilant to the possible cause(s)and quality of the pain, and in turn, may seek clinical intervention. Stage 2 begins whenthe pain has persisted beyond a period of time that would be deemed appropriate orconducive to the healing process. It is at this stage that maladaptive thoughts and behaviors develop or, in the event that they were present in Stage 1, worsen. Thesepsychological constructs interfere with appropriate selfcare behaviors and reduce the adherence to the recommendations received from clinical interventions. Stage 3 represents the time at which chronic pain has developed due to the inadequacy of thetreatment or management of the acute pain. Further, at this stage, the pain is accompaniedby psychopathology and sociological difficulties [6].

Thought processes appear to be actively involved in the transition between acute andchronic pain. In the initial stages of pain, the afflicted individual tends to believe that thesensation is directly associated with a particular cause or event (e.g., injury) and istemporary. However, upon the sustained presence of pain, the individual becomes awareof its permanence and adverse thoughts may develop [7]. In one study, Brox et al. [8] found that when comparing acute and chronic low back pain patients, bothgroups displayed similarities in physical performance and fear-avoidant beliefs. Thisfinding suggests that such behaviors are present in the early stages of pain and arepossibly involved in the pathogenesis from acute to chronic pain. With regard to fear avoidance, individuals may fixate on the pain, which can create an amplification of anypain stimulus that is experienced, and in turn, may discourage adequate coping and treatment-seeking behaviour [9].

From a behavioral standpoint, acute pain can be seen as progressing to become chroniconce the pain sufferer begins to seek treatment, which would indicate that the pain hasreached a level that has reached clinical importance [10]. A vast literature hasconsistently established that chronic pain can develop from the reinforcement of acutepain through operant conditioning. Feedback from the environment or from social cues may facilitate or discourage engaging in pain behaviors. For instance, if pain is elicitedfrom an activity (e.g., bending down), it is likely that such an activity will be avoided inthe future whereas secondary gains (e.g., attention from a physician) upon experience ofpain through a grimace may encourage similar behaviours [11].

\section{Psychological Comorbidity}

There is a vast literature establishing a high rate of psychological comorbidity in patientswith COP. Many of these empirical efforts have involved the administration of structured clinical interviews to ascertain the presence of present and lifetime co-existing psychological disorders. Efforts to collect data surrounding the premorbid psychological status provided investigators with a less-than-ideal means to establish whether psychological status may have served as a diathesis for patients to a particular type of pain if injury occurred in that body site. Studies citing lifetime psychiatric comorbidity has been criticized by relying excessively on patient self-reports and have often been perceived as a research ploy to circumvent a longitudinal examination of psychiatric status in a chronic pain population.

Although much data have supported a high incidence of psychological morbidity in a COP population, numerous epidemiological investigations have collectively shown thatnon-pain populations have comparable rates of psychological disorders to that of COP. Our review of these data does support a greater incidence of psychological disorders inpatients with COP; however, the type of disorder may represent a key explanation in the observed differences for sufferers with orofacial pain. Moreover, the form of COP also influences the psychosocial profile of the patient. Nifosi et al. [12] compared patients presenting with myofascial pain alone, patients with Temporomandibular Joint (TMJ) pain alone, and patients presenting with both myofascial and TMJ pain. They found that those with myofascial pain alone scored higher on several psychopathological domains compared to the other pain groups.

\section{Comorbidity of Specific Psychological Disorders and Orofacial Pain}

Anxiety disorders represent a common syndrome comprised of both emotional andpsychophysiological symptoms (i.e., muscular bracing, muscular tension, gastrointestinal distress, respiratory difficulties). There has been support for anxiety to be more commonly observed during the acute stage of pain versus the chronic one. Specifically, Gatchel et al. [13] observed higher levels of anxiety among patients with acuteTMD relative to those with chronic TMD. These and related findings underscore the notion that anxiety may represent a potential mechanism in the persistence of pain. However, other investigations have consistently found clinical levels of anxiety inchronic pain. For example, Posttraumatic Stress Disorder (PTSD) is frequently observed in patients suffering from all forms of orofacial pain [14]. Its prevalence, is believed to be the second largest to depression, and has been estimated from $10-30 \%$ in TMD [15-17]. These rates do exceed those rates observed in a non-clinical population. Moreover, patients with TMD report having experienced more traumatic events than personswithout TMD [18-20]. Gender differences have emerged from these data with females exhibiting a higher degree of TMD and PTSD symptoms compared to males. Consistent with other pain populations, the presence of PTSD in TMD increases the likelihood of a greater pain severity, lower pain threshold, poorer treatment outcomes, reduced functionality, and a higher level of disability [17,21]. Many of the aforementioned data involved patients whose traumas stem from motor vehicle accidents. In a population exposed to combat- related traumas, veterans with cooccurring PTSDand TMD reported more physical symptoms including headaches, Temporomandibular Joint (TMJ) clicking, and greater facial tenderness relative to those without PTSD [22]. Mood disorders including depression represent a common factor in chronic pain. Their presence influences numerous germane endpoints to pain patients including pain severity, pain-related disability, treatment response, and quality of life. According to Yap et al. [23,24], approximately $40 \%$ of TMD patients in their sample met criteria for clinical depression. Other studies have found that the presence of depression to range from a few symptoms of mood decline to actual full blown major depressive episodes [25]. The manner in which depression mediates and/or moderates these outcomes continues to be debated. Conditions such as TMD do not seem to present in isolation, forin one large-scale study, nearly two-thirds of patients had 3 or more comorbid conditions including depression [26]. While many conclusions from previous investigations have viewed depression as secondary to the pain, the directionality of the relationship between depression and pain remains unclear. Breslau et al. [27] found a bidirectional association between migraine and depression; specifically, each condition predicted the onset of the other condition. Although depression alone is associated with negative outcomes, the combination of depression and anxiety may pose even a greater challenge for clinicians and patients. Lithgart et al. [28] recently reported that patients with combined depression and anxiety reported a higher number of pain sites than those who presented with anxiety or depression alone. Somato form disorders are characterized by heightened somatic awareness and preoccupation, which lead to considerable impairment as well as distress. Full-blown somatoform disorders do not necessarily imply a psychogenic etiology; 
Citation: Mehalick ML, Garofalo JP, Sanders CN, Gatchel RJ (2013) Assessment of the Psychological Comorbidity, Pathophysiological Mechanisms, and Treatment Implications in Patients with Chronic Orofacial Pain. Anaplastology S2: 001. doi:10.4172/2161-1173.S2-001

Page 3 of 8

instead, their presence often indicates that psychological factors may be aggravating whatever organic factors underlie the pain condition. Further, the presence of a somatoform disorder is often indicative of patients who will resist any psychological interpretation of their symptoms. Indeed, preoccupation with any deterioration in symptoms or the prolonging of symptoms can lead to patient reports of increased pain duration, pain-related disability, and pain intensity. A significant number of studies examining psychological comorbidity have found high incidences of somatoform disorders as well as patients scoring high onsomatization scales [29].

Aside from identifying specific psychological disorders, there have been fewer efforts to measure the presence of other psychological constructs believed to influence pain symptoms. For example, stress burden has begun to receive increased attention as a pivotal variable in the manifestation and course of orofacial pain. Studies have examined differences in stress levels for patient with jaw pain to those with other forms of pain (e.g., low back pain, fibromyalgia) with both selfreport and physiological measures. Central to this work has been the enhanced understanding of endocrine measures ofstress, including but not limited to the Hypothalamic-Pituitary-Adrenal axis (HPA).

Pain catastrophizing is a construct defined as a tendency to magnify, ruminate, and experience helplessness in response to pain [30]. To date, the catastrophizing of pain has been shown to be one of the most robust predictors of pain outcomes. In part, the influence of catastrophizing has been associated with heightened activation of neural regions believed to influence the emotional components of pain. In one exquisitely sophisticated design, investigators found that high pain catastrophizers reported increased pain when experiencing high threat than when experiencing low threat as a result of bothaffective and cognitive mechanisms that assist in the processing of both threat and pain [31].

Collectively, the data establishing a robust association between orofacial pain and psychological comorbidity has lead to the question of whether treatment approaches need to be reformulated to adequately address this form of pain. Further, it does underscore the potential etiological pathways in which pain emerges. For example, the presence of psychological distress may trigger muscular activity that eventually might elicit pain via mechanical pathways. The documented psychological comorbidity also allows us to speculate that the processing of pain has been compromised by the same abnormal neurotransmitter imbalances observed in psychological disorders [32]. A sharedbiological substrate bolsters the argument that orofacial pain is an expression of underlying psychological distress (i.e., depression and/or anxiety).

\section{Nociceptive Transmission of Orofacial Pain}

The orofacial region is innervated by the trigeminal nerve (i.e., cranial nerve V), whichbranches into three divisions; the ophthalmic, extending to the infra-orbital area, the maxillary, surrounding the nasal region and above the mouth, and the mandibular, including the tongue, teeth, oral mucosa, and muscles of mastication $[33,34]$. Otherprimary afferent nerves such as $\mathrm{C}$ fibers, $\mathrm{A}^{\mathrm{m}}$, and $\mathrm{A}^{\circ}$ fibers also innervate the orofacial region and are responsible for encoding sensory inputs such as pain, temperature changes, tactile, and mechanical stimulation. During acute pain such as when a painful stimulus reaches the face, primary afferent neurons transmit nociceptive inputs from the orofacial area to the trigeminal ganglion, and then project into the brainstem and spinal cord, and terminate in the cerebral cortex where the affective and discriminative aspects of pain are processed. When pain becomes chronic, such as when inflammation is present or when a nerve injury occurs, this nociceptive processing pathway becomes altered via various physiological mechanisms $[35,36]$.

\section{Central and Peripheral Sensitization during Chronic Pain}

As mentioned previously, chronic pain is pain that persists beyond the healing of injuryand is often clinically defined as pain lasting longer than 6 months. Although chronic pain affects various parts of the body and can be inflammatory or neuropathic in nature, several common physiological changes in pain processing neurons occur among chronic pain conditions that are responsible for maintaining the pain. When pain becomes chronic, the term "sensitization" is often used as an umbrella term to describe the many specific changes within the cells and anatomical pathways that process nociception. Sensitization refers to an increase or enhancement of neuronal activity and occurs both centrally and peripherally to maintain pain. Further, the neural mechanisms underlying sensitization are the main factors distinguishing chronic pain from acute pain. Much like other forms of chronic pain, chronic orofacial pain is characterized by several broad mechanisms of sensitization that contribute to ongoing pain. First, primary afferent neurons become hyperactive in transmitting nociceptive sensory inputs to the spinal cordand brain, and this hyperactivity is facilitated by the release of various neurotransmitters and neuromodulators. Second, the major excitatory and inhibitory neurotransmitters suchas glutamate and GABA that facilitate and inhibit nociceptive inputs become"unbalanced," and sufficient pain inhibition mechanisms become impaired. Third, nociceptive neurons undergo changes within their firing thresholds and membrane excitability, which alter intracellular signalling pathways and produce functional changes. Lastly, phenotypic changes occur in primary afferent neurons that change their responseto various sensory stimuli [37-39]. Together, these events create and maintain a state of peripheral and central sensitization that governs chronic pain conditions.

\section{Trigeminal Brainstem Nuclear Sensory Complex (VBSNC) and Orofacial Pain}

Chronic orofacial pain shares many of the same major pathophysiological changes withinnociceptive processing neurons as other forms of chronic pain. However, chronic orofacial pain does appear to differ in terms of where, anatomically, the majority of the nociceptive inputs are processed. Orofacial pain is unique in that the central mechanisms for nociceptive processing occur not only in the spinal cord but also in a specific region of the brainstem. This region is known as the trigeminal brainstem nuclear sensorycomplex (VBSNC), or simply, the trigeminal sensory nucleus. Anatomically, this bilateral region is located dorsolateral within the brainstem and contains a main sensory nucleus and three subnuclei; the nucleus oralis (Vo), the nucleus interpolaris $(\mathrm{Vi})$, and the nucleus caudalis $(\mathrm{Vc})[34,40]$.

The subnuclei within the VBSNC, in particular the nucleus caudalis, are responsible fororofacial nociceptive processing and share some similar anatomical and physiological properties with spinal dorsal horn neurons [41,42] (Table 1 and 2). For this reason, theVBSNC is also known as the "medullary dorsal horn." However, of important note is that the caudal portion of the nucleus caudalis extends anatomically to the top portion of the spinal cord. Given this anatomical proximity, there is likely some overlap of orofacial nociceptive processing between the nucleus caudalis and spinal cord. Nevertheless, the caudalis region of the VBSNC is considered a separate anatomical region with 


\begin{tabular}{|c|}
\hline Physiological Characteristics: \\
\hline Neurotransmitters \\
\hline Glutamate \\
\hline GABA \\
\hline Glycine \\
\hline Substance P \\
\hline Neuropeptide $Y$ \\
\hline Serotonin $(5-\mathrm{HT})$ \\
\hline Kainate \\
\hline Receptors: \\
\hline mGluR1, mGluR2, \& mGluR3 \\
\hline NRB1 \& NRB2 A/B \\
\hline GABAA/B \\
\hline P2X3-7 \\
\hline TRPA1 \\
\hline TRPV1 \\
\hline NK1 \& NK2 \\
\hline AMPA \\
\hline KA \\
\hline Neuromodulators: \\
\hline Calcitonin gene-related peptide (CGRP) \\
\hline Brain-derived neurotrophic factor \\
\hline (BDNF) \\
\hline Neurokinin-1 \\
\hline Purinergic \\
\hline Nitric Oxide (NO) \\
\hline Prostaglandins \\
\hline
\end{tabular}

Table 1: Mediators of sensitization in both the VBSNC and the spinal cord.

\begin{tabular}{|c|}
\hline Anatomical Characteristics \\
\hline Primary Afferents \\
\hline C fibers \\
\hline A $\delta$ fibers \\
\hline A fibers \\
Neurons \\
\hline Nociception specific (NS) \\
\hline Wide-range (WDR) \\
\hline Laminae levels \\
\hline Superficial I-III \\
\hline Deep IV-VI \\
\hline Cortical projections \\
\hline Anterior cingulate cortex (ACC) \\
\hline Somatosensory cortex \\
\hline Prefrontal cortex \\
\hline Thalamus \\
\hline Insula \\
\hline
\end{tabular}

Table 2: Anatomical similarities in nociceptive processing between VBSNC and spinaldorsal horn neurons.

specificnociceptive processing functions that differ from those of the spinal dorsal horn neurons $[43,44]$.

The subnuclei within the VBSNC are somatotopically arranged such that the neurons within each of the individual nuclei receive sensory inputs from specific regions of theface and mouth. For example, the ventral portions of the VBSNC nuclei receive inputs from the orbital region of the face, whereas the dorsal and more caudal regions receive inputs from the maxillary and mandibular facial regions [42]. Despite this somatotopic organization, there appears to be interconnections and overlap of sensory input processing among the nuclei. This overlap could partially be the result of the proximity of branching of the trigeminal nerve, along with influence from other cranial nerves such as the hypoglossal, glossopharyngeal, and vagal nerves. Additionally, the rostral portion of the nucleus caudalis connects with the caudal portion of the interpolaris nucleus and together they form what's known as a "transition region" ( $\mathrm{ViVc})$. This transition region is thought to serve as an area for extensive overlap of nociceptive inputs from the face and mouth, such as within the corneal areas and masseter muscle, and primarily from theophthalmic division of the trigeminal nerve [4447]. The nucleus caudalis has also been shown to independently receive nociceptive inputs while also activating the $\mathrm{ViVc}$ transition region, suggesting that both areas are responsive to incoming nociception [4750]. Such extensive peripheral innervation from cranial nerves along with overlap ofcentral innervation within the $\mathrm{ViVc}$ and nucleus caudalis may suggest a state of "overrepresentation" of orofacial nociceptive inputs. From a physiological standpoint, this overrepresentation may partially explain the severity and chronicity of orofacial pain conditions [47] compared to other chronic pain conditions.

Many other forms of chronic pain such as low back pain or joint pain differ in that the nociceptive processing of sensory inputs occurs predominately in the dorsal horn region of the spinal cord. In contrast to the VBSNC, the spinal cord dorsal region is one single area of sensory innervation from both the periphery and among neurons within the dorsal horn. The spinal dorsal horn region is layered, or laminated, meaning that the cells are within different anatomical locations beginning with the superficial layers (i.e. laminae IIII) and extending down into deeper layers (i.e. laminaeIV-V1). Given this organization, interneurons and incoming primary afferents converge within multiple layers and thus are subjected to extensive sensory input. The nucleus caudalis in the VBSNC is also laminated similarly to the spinal dorsal horn area, whereas the $\mathrm{ViVc}$ transition region and other VBSNC nuclei are not [44]. Although the lamination organization is similar, is it possible that the wide spread sensory innervation of the nucleus caudalis sets the stage forenhanced central sensitization for orofacial pain states compared to other chronic pain states.

Despite some differences in sensory innervation, the VBSNC and spinal cord do share similar anatomical ascending and descending projections that are involved with nociceptive processing. For example, the VBSNC also projects to adjacent brainstem areas such as the reticular formation, the cerebellum, the pontine brachical nucleus, the Periaqueductal Gray (PAG), the Rostral Ventromedial Medulla (RVM), and to the cervical region of the spinal cord. Both the PAG and RVM are regions responsible for the descending inhibition of nociceptive inputs within the spinal dorsal neurons, and havealso been shown to influence nociceptive inputs to the nucleus caudalis. Much like spinalcord neurons, neural projections within the VBSNC also ascend to the thalamus and tovarious cortical areas such as the somato sensory cortex, anterior cingulate cortex, insula, and prefrontal cortex, where various sensory and affective dimensions of pain are processed $[42,43]$. Given these similarities, certain aspects of orofacial pain such as affective aspects, may be similar to other chronic pain conditions.

\section{Mechanisms of Sensitization within the VBSNC}

The VBSNC contains Nociceptive Specific (NS) neurons, or neurons that encode noxiousstimuli, along with Wide Range Neurons (WDR), which encode a broad spectrum of stimuli ranging from slight tactile stimuli to more pronounce mechanical and painfulstimuli [51] 
Citation: Mehalick ML, Garofalo JP, Sanders CN, Gatchel RJ (2013) Assessment of the Psychological Comorbidity, Pathophysiological Mechanisms, and Treatment Implications in Patients with Chronic Orofacial Pain. Anaplastology S2: 001. doi:10.4172/2161-1173.S2-001

Page 5 of 8

During acute pain, both types of neurons are active at transmitting nociceptive and non-nociceptive inputs. However, as pain becomes chronic, both types of neurons become more sensitive and less discriminative of sensory stimuli. When a nerve injury or inflammation persists, both the NS and WDR neurons become more active; thatis, the membrane excitability increases, and the thresholds for firing decrease. Evidence of hyperactivity in NS and WDR neurons has been found in electrophysiological studies showing that the ectopic firing patterns of these neurons increase, along with the post firing discharge [52-57]. The sensitivity and phenotype of primary afferents also changes such that $A^{\star}$ fibers that typically respond to tactile and lighter pressure stimulation, now respond to noxious stimulation. Once the discriminatory abilities and the firing thresholds of these neurons weaken, a greater amount of sensory inputs are processed and in turn contribute to increased excitation, or facilitation of nociceptive inputs. Further, as pain becomes chronic, primary afferents undergo changes in plasticity, or synaptic reorganization that facilitates nociceptive processing [58]. For example, several adjacent neurons will form new synaptic connections onto a single neuron, which allows for increased convergence of nociceptive inputs. Plasticity changes also involve new "sprouting" of neurons, often within the periphery that further facilitate central processing of nociceptive sensory inputs. Orofacial nerve injuries are associated with sprouting of sensory afferents peripherally and appear to maintain pain, yet sprouting within the trigeminal ganglion appears to be spared $[56,59]$.

Central sensitization involves physiological changes in pain processing such that nociceptive inputs are facilitated more than inhibited. Under conditions of acute pain, nociceptive facilitation and inhibition are in equilibrium, where afferent nociceptive inputs are adequately inhibited by efferent outputs. Descending inhibitory supraspinal-structures such as the Periaqueductal Gray (PAG) and Rostral Ventromedial Medulla (RVM) send descending projections to inhibit incoming nociceptive inputs within the spinal cord [60,61]. However, when pain becomes chronic, the equilibrium becomes"unbalanced" and nociceptive input outweighs nociceptive inhibition [62]. Given that theVBSNC is part of the brainstem; this region is not exempt from the imbalance of nociceptive facilitation and inhibition. For example, the $\mathrm{ViVc}$ transition region and theRVM have bidirectional projections and following temporomadibular injury orinflammation, the RVM neurons have been shown to facilitate nociceptive processing within the $\mathrm{ViVc}$ region $[63,64]$. In addition to peripheral inputs influencing nociceptive facilitation, various neurotransmitters and neuromodulators are released during chronic pain that exacerbate facilitation [34]. Like spinal neurons, neurons within the VBSN Crelease excess glutamate, causing neurons to become more active, and hence more efficient at transmitting nociceptive signals to the brain. During nerve injury or inflammation to the orofacial region primary afferents, including the trigeminal nerve, release neurotransmitters and neuromodulators such as substance P, Brain-Derived Neurotrophic Factor (BDNF), and Calcitonin Gene-Related Peptide (CGRP) also facilitate nociceptive transmission. In contrast, inhibitory neurotransmitters such as GABA and glycine cannot adequately inhibit the influx of nociceptive inputs [65]. Evidence for this imbalance comes from animal studies where GABA agonists such as baclofen are administered into the dorsal horn spinal neurons, and pain behavior decreases, suggesting that increasing inhibitory tone during chronic pain helps with restoring tonic inhibition [66]. Various other physiological factors are responsible for maintaining central and peripheral sensitization and include but are not limited to neurotransmitters, neuromodulators, receptor subtypes, changes in intercellular signalling proteins, and chemical mediators such as cytokines and chemokines.

\section{Cellular Mechanisms of Sensitization}

During chronic orofacial pain various receptors act peripherally and centrally within the VBSNC to initiate the development and maintenance of pain $[34,67]$. Excitation of primary trigeminal afferents occur through ionotropic calcium and sodium ion-gated channels within cell membranes, and become depolarized when excitatory neurotransmitters such as glutamate bind to NMDA receptor subtypes NR2A/B [68]. AMPA receptors, specifically the GluR2 and GluR3 subtypes have also been shown toincrease neural excitation within the nucleus caudalis and upper cervical spinal neuronsupon injury to the trigeminal nerve [69]. Purinergic receptor subtypes P2X3/7 have also been shown to facilitate both peripheral sensitization among trigeminal nerve afferents, and also central sensitization among neurons within the caudalis region of the VBSNC [56,70-72]. Much like spinal neurons of the dorsal root ganglion, trigeminal ganglion neurons show expression of the transient receptor potential vanilloid 1 (TRPV1) following trigeminal nerve injury or experimentally applied capsaicin $[73,74]$. Pain resulting from tooth pulp inflammation is mediated by the transient receptor potential ankyrin repeat 1 (TRPA1) ionotrophic receptor, a subtype of the TRPV1. The TRPA1 receptor also becomes activated by various chemical irritants, cannabinoids, and coldstimuli [75]. During inflammatory chronic orofacial pain conditions, Nerve Growth Factor (NGF) and other inflammatory mediators such as ILfacilitate the upregulation of TRPA1 receptors [75]. Depolarization of primary nociceptors also leads to functional changes among $\mathrm{G}$ proteins and metabotrophic receptors within the neuron. Upon activation, $G$ proteins can produce either excitation or inhibition of specific intracellular pathways. Intracellular pathways involve the activation of various protein kinases and messenger proteins that in turn influence cellular function. Several intracellular pathways have been identified as targets for maintaining central sensitization, including protein

Kinase C (PKC), Protein Kinase A (PKA), Extracellular SignalRelated Kinase (ERK), and p38 Mitogen-Activated Protein Kinase (MAPK) $[74,76,77]$. Many of these intracellular proteins are activated by surface membrane receptors; specifically receptors for glutamate (mGluR), neurokinin (NK1), and calcitonin gene-related peptide (CGRP1), which allactivate the ERK pathway. Within the VBSNC, ERK activation has been shown in the caudalis region following both trigeminal nerve injuries and during conditions of dentalinflammation [77-79]. These various signalling pathways may partially explain thediversity and complexity of orofacial pain conditions.

\section{Glial Mechanisms of Sensitization}

During orofacial pain, in particular, following injuries to the trigeminal nerve branches, glia cells have been shown to facilitate the maintenance of cellular mechanisms of central sensitization within the VBSNC $[76,80]$. Microglias surrounds and structurally "support"primary afferent neurons within the periphery around the dorsal root ganglion, trigeminalganglion, and centrally within the spinal cord and VBSNC. Microglia and neurons are connected by gap-like junctions that promote the exchange of neurotransmitters, neuromodulators, and chemical mediators. Microglias are thought to further contribute tocentral sensitization by facilitating the release of excitatory neurotransmitters from neurons [80]. For example, excitatory neurotransmitters such as glutamate and kainite enhance the excitability of the gap junctions among microglia via influx of calcium ions, thus causing depolarization of neurons. Additionally, in response to inflammation orinjury of the trigeminal nerve, microglia release substance P, Nitric Oxide (NO), neurokinin, and prostaglandins, which 
Citation: Mehalick ML, Garofalo JP, Sanders CN, Gatchel RJ (2013) Assessment of the Psychological Comorbidity, Pathophysiological Mechanisms, and Treatment Implications in Patients with Chronic Orofacial Pain. Anaplastology S2: 001. doi:10.4172/2161-1173.S2-001

Page 6 of 8

together enhance neuron excitability within the nucleus caudalis of the VBSNC $[78,81,82]$. During injury of the trigeminal nerve, microglia have also been shown to release proinflammatory cytokines such as IL- ${ }^{-}$andTNF, and chemokines such as CCL2, which contribute to central sensitization of neurons within the VBSNC, particularly following inflammation [71,83-85]. Astroglia areanother type of glial cell that further maintain chronic orofacial pain. Like neurons, astroglia have receptors for glutamate (e.g. NMDA) substance P, and GABA, which make them sensitive to changes in excitatory and inhibitory neurotransmitter release during sensitization. Specifically, when mechanisms of central sensitization develop, astroglia appear to function as "cleaner cells" by eliminating excess glutamate via reuptakemechanisms. Thus, astroglia are also thought to serve as a means of dampening central sensitization.

\section{Conclusion}

Categorical efforts to identify, assess, and treat orofacial pain is limited by a certain degree of heterogeneity within this pain population. Indeed, the highlighting of specific mechanisms does imply that we propose a definitive pathophysiological model of orofacial pain. Instead, the intention is to identify distinct mechanisms in which COP may manifest. Future studies should focus on the role of the physiological mechanisms and the manner in which they work in concert with psychosocial variables to influence the course of COP and the response of patients to treatment interventions. In conclusion, the mechanisms identified here that distinguish chronic orofacial pain from other pain populations provide testable hypotheses concerning its development and course. Continued efforts to empirically investigate these questions in COP should focus on furthering a guiding conceptual frame work that can include some of the understudied psychophysiological variables. With an enhanced understanding of this population's psychological and psychophysiological landscape, a greater need for more tailored interventions further emerges. Ultimately, the testing of such a frame work would allow researchers to determine whether the convergence of factors suspected in orofacial pain can lead to better integrative interventional approaches to treat a pain condition often times perceived as treatment reticent. Within that same context, investigators should also examine whether tailored treatments actually yield different results from more "generic"approaches.

\section{Acknowledgements}

We thank the Journal of Anaplastology for the invitation of this manuscript.

\section{References}

1. Scrivani SJ, Keith DA, Kaban LB (2008) Temporomandibular disorders. N Engl J Med 359: 2693-2705.

2. Nagarajan M, Nair MR (2010) Importance of fear-avoidance behavior in chronic non-specific low back pain. J Back MusculoskeletRehabil 23: 87-95.

3. Vickers ER, Cousins MJ, Woodhouse A (1998) Pain description and severity of chronic orofacial pain conditions. Aust Dent J 43: 403-409.

4. Ashburn MA, Staats PS (1999) Management of chronic pain. Lancet 353: 1865 1869.

5. Epker J, Gatchel RJ, Ellis E 3rd (1999) A model for predicting chronic TMD: practical application in clinical settings. J Am Dent Assoc 130: 1470-1475.

6. Gatchel RJ, Bernstein D, Stowell AW, Pransky G (2008) Psychosocial differences between high-risk acute vs. chronic low back pain patients. Pain Pract 8: 91-97.

7. Barowsky El (1987) Factors affecting the impact of pain and painful experienceson the exceptional child. Journal of Special Education 21: 109-121.

8. Brox JI, Storheim K, Holm I, Friis A, Reikerås O (2005) Disability, pain, psychological factors and physical performance in healthy controls, patients with sub-acute and chronic low back pain: a case-control study. J Rehabil Med 37: 95-99.

9. Linton SJ, Buer N, Vlaeyen J, Hellsing AL (2000) Are fear-avoidance beliefs related to the inception of an episode of back pain? A prospective study. Psychol Health 14: 1051-1059.

10. Garofalo JP, Gatchel RJ, Wesley AL, Ellis E 3rd (1998) Predicting chronicity in acute temporomandibular joint disorders using the research diagnostic criteria. J Am Dent Assoc 129: 438-447.

11. Stiles TC, Wright D (2008) Cognitive-behavioural treatment of chronic pain conditions. Nord J Psychiatry 62 Suppl 47: 30-36.

12. Nifosì F, Violato E, Pavan C, Sifari L, Novello G, et al. (2007) Psychopathology and clinical features in an Italian sample of patients with myofascial and temporomandibular joint pain: preliminary data. Int J Psychiatry Med 37: 283300

13. Gatchel RJ, Garofalo JP, Ellis E, Holt C (1996) Major psychological disorders in acute and chronic TMD: an initial examination. J Am Dent Assoc 127: 1365$1370,1372,1374$

14. Blanchard EB, Hickling EJ, Taylor AE, Loos W (1995) Psychiatric morbidity associated with motor vehicle accidents. J NervMent Dis 183: 495-504.

15. Aghabeigi B, Feinmann C, Harris M (1992) Prevalence of post-traumatic stress disorder in patients with chronic idiopathic facial pain. $\mathrm{Br} \mathrm{J}$ Oral MaxillofacSurg 30: $360-364$.

16. Carlson CR, Reid KI, Curran SL, Studts J, Okeson JP, et al. (1998) Psychologica and physiological parameters of masticatory muscle pain. Pain 76: 297-307.

17. Sherman JJ, Carlson CR, Wilson JF, Okeson JP, McCubbin JA (2005) Posttraumatic stress disorder among patients with orofacial pain. J Orofac Pain 19: 309-317

18. Afari N, Wen Y, Buchwald D, Goldberg J, Plesh O (2008) Are posttraumaticstress disorder symptoms and temporomandibular pain associated? Findings froma community-based twin registery. J Orofac Pain 22: 41-49.

19. De Leeuw R, Bertoli E, Schmidt JE, Carlson CR (2005) Prevalence of traumatic stressors in patients with temporomandibular disorders. J Oral MaxillofacSurg 63: 42-50.

20. De Leeuw R, Bertoli E, Schmidt JE, Carlson CR (2005) Prevalence of posttraumatic stress disorder symptoms in orofacial pain patients. Oral Surg Oral Med Oral Pathol Oral Radiol Endod 99: 558-568

21. Speculand B, Hughes AO, Goss AN (1984) Role of recent stressful life events experience in the onset of TMJ dysfunction pain. Community Dent Oral Epidemiol 12: 197-202.

22. Uhac I, Kovac Z, MuhviÄ -Urek M, KovaceviÄ $D$, FranciskoviÄ $T$, et al (2006) The prevalence of temporomandibular disorders in war veterans with post-traumatic stress disorder. Mil Med 171: 1147-1149.

23. Yap AU, Chua EK, Dworkin SF, Tan HH, Tan KB (2002) Multiple pains and psychosocial functioning/psychologic distress in TMD patients. Int Prosthodont 15: 461-466.

24. Yap AU, Chua EK, Hoe JK (2002) Clinical TMD, pain-related disability and psychological status of TMD patients. J Oral Rehabil 29: 374-380.

25. Hestbaek L, Leboeuf-Yde C, Manniche C (2003) Is low back pain part of a general health pattern or is it a separate and distinctive entity? A critical literature review of comorbidity with low back pain. J Manipulative PhysiolTher 26: $243-252$

26. Hoffmann RG, Kotchen JM, Kotchen TA, Cowley T, Dasgupta M, et al. (2011) Temporomandibular disorders and associated clinical comorbidities. Clin J Pain 27: $268-274$

27. Breslau N, Lipton RB, Stewart WF, Schultz LR, Welch KM (2003) Comorbidity of migraine and depression: investigating potential etiology and prognosis. Neurology 60: 1308-1312.

28. Ligthart L, Gerrits MM, Boomsma DI, Penninx BW (2013) Anxiety and depression are associated with migraine and pain in general: an investigation of the interrelationships. J Pain 14: 363-370.

29. Komiyama O, Obara R, Uchida T, Nishimura H, lida T, et al. (2012) Pain intensity and psychosocial characteristics of patients with burning mouth syndrome and trigeminal neuralgia. J Oral Sci 54: 321-327. 
Citation: Mehalick ML, Garofalo JP, Sanders CN, Gatchel RJ (2013) Assessment of the Psychological Comorbidity, Pathophysiological Mechanisms, and Treatment Implications in Patients with Chronic Orofacial Pain. Anaplastology S2: 001. doi:10.4172/2161-1173.S2-001

Page 7 of 8

30. Sullivan MJ, Thorn B, Haythornthwaite JA, Keefe F, Martin M, et al. (2001) Theoretical perspectives on the relation between catastrophizing and pain. Clin J Pain 17: 52-64.

31. Peeters PA, Vlaeyen JW (2011) Feeling more pain, yet showing less: the influence of social threat on pain. J Pain 12: 1255-1261.

32. Kindler S, Samietz S, Houshmand M, Grabe HJ, Bernhardt O, et al. (2012) Depressive and anxiety symptoms as risk factors for temporomandibular joint pain: a prospective cohort study in the general population. J Pain 13: 11881197.

33. Maciewicz R (1990) Neurologic aspects of chronic facial pain. AnesthProg 37 129-132.

34. Sessle BJ (2011) Peripheral and central mechanisms of orofacial inflammatory pain. Int Rev Neurobiol 97: 179-206.

35. Obermann M, Yoon MS, Ese D, Maschke M, Kaube H, et al. (2007) Impaired trigeminal nociceptive processing in patients with trigeminal neuralgia. Neurology 69: 835-841.

36. Sessle BJ (2002) Recent insights into brainstem mechanisms underlying craniofacial pain. J Dent Educ 66: 108-112.

37. Millan MJ (1999) The induction of pain: an integrative review. ProgNeurobio 57: $1-164$.

38. Graff-Radford SB (2009) Facial pain. Neurologist 15: 171-177.

39. Miki K, Zhou QQ, Guo W, Guan Y, Terayama R, et al. (2002) Changes in gene expression and neuronal phenotype in brain stem pain modulatory circuitry after inflammation. J Neurophysiol 87: 750-760.

40. Bae YC, Yoshida A (2011) Ultrastructural basis for craniofacial sensory processing in the brainstem. Int Rev Neurobiol 97: 99-141.

41. Park SJ, Zhang S, Chiang CY, Hu JW, Dostrovsky JO, et al. (2006) Centra sensitization induced in thalamic nociceptive neurons by tooth pulp stimulation is dependent on the functional integrity of trigeminal brainstem subnucleuscaudalis but not subnucleusoralis. Brain Res 1112: 134-145.

42. Sessle BJ (2000) Acute and chronic craniofacial pain: brainstem mechanisms of nociceptive transmission and neuroplasticity, and their clinical correlates. Crit Rev Oral Biol Med 11: 57-91.

43. Sessle BJ (2005) Peripheral and central mechanisms of orofacial pain and their clinical correlates. Minerva Anestesiol 71: 117-136.

44. Bereiter DA, Hirata H, Hu JW (2000) Trigeminalsubnucleuscaudalis: beyond homologies with the spinal dorsal horn. Pain 88: 221-224.

45. Arvidsson J, Pfaller K, Gmeiner S (1992) The ganglionic origins and central projections of primary sensory neurons innervating the upper and lower lips in the rat. Somatosens Mot Res 9: 199-209.

46. Capra NF, Dessem D (1992) Central connections of trigeminal primary afferent neurons: topographical and functional considerations. Crit Rev Oral Biol Med 4: $1-52$.

47. Ren K, Dubner R (2011) The role of trigeminal interpolaris-caudalis transition zone in persistent orofacial pain. Int Rev Neurobiol 97: 207-225.

48. Shimizu K, Guo W, Wang H, Zou S, LaGraize SC, et al. (2009) Differential involvement of trigeminal transition zone and laminated subnucleuscaudalis in orofacial deep and cutaneous hyperalgesia: the effects of interleukin-10 and glial inhibitors. Mol Pain 5: 75 .

49. Chang Z, Okamoto K, Tashiro A, Bereiter DA (2010) Ultraviolet irradiation ofthe eye and Fos-positive neurons induced in trigeminal brainstem after intravitrealor ocular surface transient receptor potential vanilloid1 activation. Neuroscience170: 678-685

50. Voisin DL, Doméjean-Orliaguet S, Chalus M, Dallel R, Woda A(2002)Ascending connections from the caudal part to the oral part of the spinal trigeminal nucleus in the rat. Neuroscience 109: 183-193.

51. Hu JW, Sessle BJ, Raboisson P, Dallel R, Woda A (1992) Stimulation of craniofacial muscle afferents induces prolonged facilitatory effects in trigeminal nociceptive brain-stem neurones. Pain 48: 53-60

52. Kitagawa J, Takeda M, Suzuki I, Kadoi J, Tsuboi Y, et al. (2006) Mechanisms involved in modulation of trigeminal primary afferent activity in rats with peripheral mononeuropathy. Eur J Neurosci 24: 1976-1986.

53. Martin YB, Malmierca E, Avendaño C, Nuñez A (2010) Neuronal disinhibition in the trigeminal nucleus caudalis in a model of chronic neuropathic pain. Eur J Neurosci 32: 399-408.

54. Eriksson J, Jablonski A, Persson AK, Hao JX, Kouya PF, et al. (2005) Behaviora changes and trigeminal ganglion sodium channel regulation in an orofacia neuropathic pain model. Pain 119: 82-94.

55. Davies SL, Loescher AR, Clayton NM, Bountra C, Robinson PP, et al. (2006) Changes in sodium channel expression following trigeminal nerve injury. ExpNeurol 202: 207-216.

56. Fried K, Bongenhielm U, Boissonade FM, Robinson PP (2001) Nerve injuryinduced pain in the trigeminal system. Neuroscientist 7: 155-165.

57. Coste J, Voisin DL, Miraucourt LS, Dallel R, Luccarini P (2008) Dorsal horn NK1-expressing neurons control windup of downstream trigeminal nociceptive neurons. Pain 137: 340-351.

58. Yu XM, Sessle BJ, Hu JW (1993) Differential effects of cutaneous and deep application of inflammatory irritant on mechanoreceptive field properties of trigeminal brain stem nociceptive neurons. J Neurophysiol 70: 1704-1707.

59. Grelik C, Bennett GJ, Ribeiro-da-Silva A (2005) Autonomic fibre sprouting and changes in nociceptive sensory innervation in the rat lower lip skin following chronic constriction injury. Eur J Neurosci 21: 2475-2487.

60. Carlson JD, Maire JJ, Martenson ME, Heinricher MM (2007) Sensitization of pain-modulating neurons in the rostral ventromedial medulla after peripheral nerve injury. J Neurosci 27: 13222-13231.

61. Bee LA, Dickenson AH (2007) Rostral ventromedial medulla control of spinal sensory processing in normal and pathophysiological states. Neuroscience 147: 786-793.

62. Porreca F, Ossipov MH, Gebhart GF (2002) Chronic pain and medullary descending facilitation. Trends Neurosci 25: 319-325.

63. Zhou Q, Imbe H, Dubner R, Ren K (1999) Persistent Fos protein expression after orofacial deep or cutaneous tissue inflammation in rats: implications for persistent orofacial pain. J Comp Neurol 412: 276-291.

64. Okubo M, Castro A, Guo W, Zou S, Ren K, et al. (2013) Transition to persisten orofacial pain after nerve injury involves supraspinal serotonin mechanisms. J Neurosci 33: 5152-5161.

65. Zeilhofer HU, Zeilhofer UB (2008) Spinal dis-inhibition in inflammatory pain NeurosciLett 437: 170-174.

66. Munro G, Ahring PK, Mirza NR (2009) Developing analgesics by enhancing spinal inhibition after injury: GABAA receptor subtypes as novel targets. Trends PharmacolSci 30: 453-459.

67. Sessle BJ (2006) Mechanisms of oral somatosensory and motor functions and their clinical correlates. J Oral Rehabil 33: 243-261.

68. Latremoliere A, Woolf CJ (2009) Central sensitization: a generator of pain hypersensitivity by central neural plasticity. J Pain 10: 895-926.

69. Miyamoto M, Tsuboi Y, Takamiya K, Huganir RL, Kondo M, et al. (2011) Involvementof GluR2 and GluR3 subunit C-termini in the trigeminal spinal subnucleuscaudalis and $\mathrm{C} 1-\mathrm{C} 2$ neurons in trigeminal neuropathic pain Neuroscience Letters491: 8-12.

70. Cherkas PS, Dostrovsky JO, Sessle BJ (2012) Activation of peripheral P2X receptors is sufficient to induce central sensitization in rat medullary dorsal horn nociceptive neurons. Neurosci Lett 526: 160-163.

71. Murasaki K, Watanabe M, Takahashi K, Ito G, Suekawa Y, et al. (2013) P2X receptor and cytokines contribute to extra-territorial facial pain. J Dent Res 92 260-265.

72. Ambalavanar R, Dessem D (2009) Emerging peripheral receptor targets for deep-tissue craniofacial pain therapies. J Dent Res 88: 201-211.

73. Kim HY, Park CK, Cho IH, Jung SJ, Kim JS, et al. (2008) Differential Changes in TRPV1 expression after trigeminal sensory nerve injury. J Pain 9: 280-288.

74. Honda K, Kitagawa J, Sessle BJ, Kondo M, Tsuboi Y, et al. (2008) Mechanisms involved in an increment of multimodal excitability of medullary and uppe cervical dorsal horn neurons following cutaneous capsaicin treatment. Mol Pain 4: 59

75. Diogenes A, Akopian AN, Hargreaves KM (2007) NGF up-regulates TRPA1: implications for orofacial pain. J Dent Res 86: 550-555. 
Citation: Mehalick ML, Garofalo JP, Sanders CN, Gatchel RJ (2013) Assessment of the Psychological Comorbidity, Pathophysiological Mechanisms, and Treatment Implications in Patients with Chronic Orofacial Pain. Anaplastology S2: 001. doi:10.4172/2161-1173.S2-001

76. Piao ZG, Cho IH, Park CK, Hong JP, Choi SY, et al. (2006) Activation of glia and microglial p38 MAPK in medullary dorsal horn contributes to tactile hypersensitivity following trigeminal sensory nerve injury. Pain 121: 219-231.

77. Patil CS, Kirkwood KL (2007) p38 MAPK signaling in oral-related diseases. J Dent Res 86: 812-825.

78. Shibuta K, Suzuki I, Shinoda M, Tsuboi Y, Honda K, et al. (2012) Organization of hyperactive microglial cells in trigeminal spinal subnucleuscaudalis and upper cervical spinal cord associated with orofacial neuropathic pain. Brain Res 1451: 74-86.

79. Suzuki I, Tsuboi Y, Shinoda M, Shibuta K, Honda K, et al. (2013) Involvement of ERK phosphorylation of trigeminal spinal subnucleuscaudalis neurons in thermal hypersensitivity in rats with infraorbital nerve injury. PLoS One 8 : e57278.

80. Chiang CY, Dostrovsky JO, Iwata K, Sessle BJ (2011) Role of glia in orofacial pain. Neuroscientist 17: 303-320.
81. Xu M, Aita M, Chavkin C (2008) Partial infraorbital nerve ligation as a model of trigeminal nerve injury in the mouse: behavioral, neural, and glial reactions. $J$ Pain 9: 1036-1048.

82. Xie YF, Zhang S, Chiang CY, Hu JW, Dostrovsky JO, et al. (2007) Involvement of glia in central sensitization in trigeminal subnucleuscaudalis (medullary dorsal horn). Brain Behavlmmun 21: 634-641.

83. Zhang ZJ, Dong YL, Lu Y, Cao S, Zhao ZQ, et al. (2012) Chemokine CCL2 and its receptor CCR2 in the medullary dorsal horn are involved in trigeminal neuropathic pain. J Neuroinflammation 9: 136

84. Guo W, Wang H, Watanabe M, Shimizu K, Zou S, et al. (2007) Glial-cytokineneuronal interactions underlying the mechanisms of persistent pain. J Neurosci 27: $6006-6018$

85. Anderson LC, Rao RD (2001) Interleukin-6 and nerve growth factor levels in peripheral nerve and brainstem after trigeminal nerve injury in the rat. Arch Oral Biol 46: 633-640

Canada. 